\title{
Persistently Gadolinium-Enhancing Lesion Is a Predictor of Poor Prognosis in NMOSD Attack: a Clinical Trial
}

\author{
Yun $\mathrm{Xu}^{1} \cdot \mathrm{Yi} \mathrm{Ren}^{2} \cdot$ Xindi $\mathrm{Li}^{1} \cdot$ Wangshu Xu ${ }^{1} \cdot$ Xinli Wang ${ }^{3} \cdot$ Yunyun Duan ${ }^{4} \cdot$ Yaou Liu ${ }^{4} \cdot$ Xinghu Zhang ${ }^{1}$. \\ De-Cai $\operatorname{Tian}^{1,5}$ (1)
}

Accepted: 13 November 2020 / Published online: 19 January 2021

(C) The American Society for Experimental NeuroTherapeutics, Inc. 2021

\begin{abstract}
Gadolinium (Gd)-contrast MRI for reliable detection of blood-brain barrier (BBB) breakdown is widely used in neuromyelitis optica spectrum disorder (NMOSD) attack. Nonetheless, little is known about the predictive role of gadolinium-enhancing lesion in prognosis of NMOSD attack. The aim of this work is to investigate the predictive value of persistently Gd-enhanced lesions to medium-term outcome after attack. Data for this analysis came from an ongoing prospective cohort study (CLUE). NMOSD patients with acute attack were enrolled from January 2019 to March 2020. All patients underwent Gd-contrast MRI at baseline and 1 month, and disability was assessed by Expanded Disability Status Scale (EDSS). Primary outcome was EDSS improvement from baseline to month 6 . Multiple logistic regression identified predictors for poor recovery of NMOSD attack. Forty-one participants were analyzed, of which 21 patients had persistently Gd-enhancing lesions. Patients in no enhancement (NE) group showed a significant shift in 6-month EDSS distributions compared with those in persistent enhancement (PE) group $(p=0.005)$. Poor recovery rate of the PE group was higher than that of the NE group at 6 months $(p=0.033)$. In patients with aquaporin-4positive, first-attack, transverse myelitis or in a high-dose steroid treatment subgroup, the improvement of EDSS scores in the PE group was still less compared with that in the NE group $(p<0.05)$. The presence of persistently Gd-enhancing lesion appears to be associated with poor recovery after attack $(\mathrm{OR}=5.473, p=0.014)$. Our study found that persistently gadolinium-enhancing lesion is a poor prognosis predictor after NMOSD attack. Trial registration ID: NCT04106830
\end{abstract}

Key Words NMOSD $\cdot$ acute attack $\cdot$ MRI $\cdot$ prognosis $\cdot$ clinical trial

Yun $\mathrm{Xu}$ and Yi Ren contributed equally to this work.

Xinghu Zhang

xhzhtiantan@hotmail.com

$\triangle$ De-Cai Tian

decaitian@hotmail.com

1 Center for Neurology, Beijing Tiantan Hospital, Capital Medical University, No. 119, South Fourth Ring Road West, Beijing 100070, China

2 Department of Neurology, Xuanwu Hospital, Capital Medical University, Beijing 100053, China

3 Department of Neurology, Tianjin Neurological Institute, Tianjin Medical University General Hospital, Tianjin 300052, China

4 Department of Radiology, Beijing Tiantan Hospital, Capital Medical University, Beijing 100070, China

5 China National Clinical Research Center for Neurological Diseases, Beijing 100070, China

\section{Introduction}

Neuromyelitis optica spectrum disorder (NMOSD) is a humoral immune-mediated astrocytic disease characterized by severe attacks of transverse myelitis and optic neuritis [1]. Aquaporin-4 antibody (AQP4-ab) as a specific marker of NMOSD has paved the way to reveal its pathogenesis [2]. Acute attacks cause severe disability and require aggressive treatment in patients with NMOSD [3]. Currently, widely recognized first-line treatments mainly include high-dose intravenous corticosteroids [4] and plasma exchange or adsorption and removal of AQP4 antibodies [5]. However, a certain proportion of patients under adequate therapy still present poor functional recovery.

In addition to known prognostic factors of NMOSD attack, such as old-age, high serum IL-6 level, high GFAP level [6-8], the breakdown of the blood-brain barrier (BBB) may also be a noteworthy factor. The BBB is formed by brain microvascular endothelial cells (BMECs) in conjunction with 
pericytes and astrocytes, which is important for maintaining brain homeostasis and preventing the entry of AQP4 autoantibodies [9]. Circulating AQP4-abs need to pass through the disrupted $\mathrm{BBB}$ in order to reach AQP4 on the astrocyte endfeet to cause the disease. It is therefore important to evaluate the BBB function and damage degree in patients with NMOSD attack.

Several methods have been developed to determinate BBB leakage. Since albumin is not produced in the central nervous system (CNS) and the BBB limits its crossover from blood to the cerebrospinal fluid (CSF), the CSF/serum albumin ratio can be used to measure disruption of the BBB. The disruption of the BBB is also characterized by the degradation of the junctional complex proteins and an increase in multiple matrix metalloproteinases [10]. MRI is a reliable method of detecting BBB breakdown in NMOSD, and gadolinium enhancement of lesions on T1-weighted MRI is interpreted as a hallmark of BBB disruption [11]. In order to understand the predictive value of post-contrast MRI persistent enhancement and provide monitoring indicators for subacute sequential treatment, we explored the clinical characteristics and medium-term prognosis of NMOSD patients after acute attack and with different MRI Gd enhancement durations.

\section{Methods}

\section{Patients}

We conducted an ongoing prospective study to assess the clinical and imaging patterns of neuroinflammation diseases in China (CLUE). All patients were enrolled between January 2019 and March 2020. NMOSD diagnosis was according to 2015 Revised International Criteria [12]. The inclusion criteria for this study were as follows: 1) aged between 15 and 75,2) present with an acute NMOSD attack, and 3) with gadolinium-enhancing lesions in the brain, spinal cord, or orbit by MRI. Acute attack was defined as a new onset of neurological symptoms which were caused by CNS damage and lasted longer than $24 \mathrm{~h}$. Exclusion criteria for the study included the following: 1) initial Expanded Disability Status Scale (EDSS) score $\geq 6$ before this attack; 2 ) treatment delay more than 3 weeks from symptom onset; 3 ) patients with heart, liver, lung, and renal failure and severe infections (e.g., HIV) and malignant tumors; 4) pregnant or nursing patients; and 5) patient had other CNS inflammatory diseases. The NMOSD patients were assigned into two groups (persistent enhancement (PE) group versus no enhancement (NE) group) according to the presence of gadolinium-enhancing lesions at 1 month.

All patients were treated with daily high-dose $(500 \mathrm{mg})$ intravenous methylprednisolone (IVMP) for five consecutive days starting on the day of diagnosis and then reduced to
$240 \mathrm{mg}$ for 3 days, followed by $120 \mathrm{mg}$ for 3 days and finally tapered orally. If clinician determined that high-dose IVMP treatment was ineffective (symptoms not improve), more intensive treatment was given. Symptom improvement is measured by Functional Systems Scale (FSS) and EDSS. Among FSS, visual functional system (VFS) is measured by Early Treatment Diabetic Retinopathy Study (ETDRS) chart (Precision Vision, Inc., Woodstock, IL) [13]. FSS same or worse than acute attack score is defined as "symptoms not improve." In addition, less than 1 step improve if attack visual acuity was worse than counting fingers also defined as "not improvement." Intravenous immunoglobulin (IVIg) was given $(0.4 \mathrm{~g} / \mathrm{kg} / \mathrm{day})$ for 5 days as further therapy, and immunoadsorption (IA) was performed every other day for a total of 5 times, using tryptophan-linked polyvinyl alcohol Immusorba TR-350 (Diamed Medizintechnik GmbH, Cologne, Germany), and treated with one plasma volume to a maximum of $3 \mathrm{~L}$ plasma per session for all patients. Physicians choose different immunosuppressive therapies based on the patient's condition: a $60 \mathrm{mg} /$ day starting dose of Prednisone, reduced $5 \mathrm{mg}$ per week; two doses of mycophenolate mofetil (MMF, $250 \mathrm{mg}$ ) daily, increased to $500 \mathrm{mg}$ after 1 week; or a course of rituximab (RTX) treatment (500 mg intravenously), repeated 2 weeks and 6 months later.

Written informed consents were provided by all participants or their guardians. This study was approved by the Ethics Committee of Beijing Tiantan Hospital, Capital Medical University, Beijing, China, and is registered with ClinicalTrials.gov (accession number NCT04106830).

\section{Clinical Assessment}

Baseline characteristics and clinical features were obtained for all recruited patients, including age at onset, gender, disease duration, serum antibody status, lesion sites, attack, and treatment profile. Physical disability was determined by an EDSS score at baseline, 1 month and 6 months after onset. All EDSS scores were assessed by trained neurologists. We calculated the proportion of EDSS score improvement: EDSS (acute 6 months post attack)/EDSS (acute - prior to attack), which ensure the comparability of interscore. Outcomes were defined as "good," "medium," and "poor recovery" representing 66-100\%, 33-66\%, and 0-33\% improvements [14], respectively. The primary assessment was to examine the association of persistent gadolinium-enhancing lesions on EDSS changes at follow-up.

\section{Laboratory Analysis}

Serum and CSF samples were collected before treatment. All samples were taken and tested at the biochemical laboratory of Beijing Tiantan Hospital. The permeability of the BBB was determined from the CSF and blood 
albumin level ratio (Qalb $=$ CSF albumin/serum albumin) [15]. Serum AQP4-IgG and MOG-IgG were test by cellbased assay (Oumeng Biotechnology Corporation, Guangzhou, China).

\section{MRI Protocol and Image Analysis}

First post-contrast MRI scans of the brain, spinal, or orbit were performed within $24 \mathrm{~h}$ from the neurologic evaluation and before treatment initiation. Second gadoliniumenhanced MRI scans were performed 4 week \pm 3 days later. All MRI scans were done on the same 3T Signa scanner (Philips Ingenia CX, Best, The Netherlands). The following MRI sequences were collected: 1) T1weighted echo $(\mathrm{TR}=7 \mathrm{~ms}, \mathrm{TE}=3 \mathrm{~ms}$, slice thickness = $1 \mathrm{~mm}$, matrix $=250 \times 250), 2) \mathrm{T} 2$-weighted echo $(\mathrm{TR}=$ $6816 \mathrm{~ms}, \mathrm{TE}=93 \mathrm{~ms}$, slice thickness $=6 \mathrm{~mm}$, matrix $=$ $512 \times 512)$, and 3 ) post-contrast ( $5 \mathrm{~min}$ after the injection of $0.1 \mathrm{mmol} / \mathrm{kg} \mathrm{Gd}) \mathrm{T} 1$-weighted echo $(\mathrm{TR}=7 \mathrm{~ms}, \mathrm{TE}=$ $3 \mathrm{~ms}$, slice thickness $=1 \mathrm{~mm}$, matrix $=250 \times 250$ ). An enhancing lesion was defined as an area with hyperintensity on T1-weighted images obtained at least 5 min after contrast agent administration, identified by consensus by two neuroradiologists who were not aware of clinical data (X.W. and Y.D.).

\section{Statistical Analyses}

Statistical analysis was conducted by SPSS 25.0 (International Business Machines Corporation, Chicago, IL). Continuous data were reported as mean \pm standard deviation or as median with interquartile range (IQR). Categorical variables were presented as absolute and relative frequencies.

Differences between the two groups (PE group versus NE group) were analyzed by the chi-square test or Fisher's exact test for categorical variables. Student's $t$ tests and Mann-Whitney $U$ tests were adopted for parametric and nonparametric continuous variables. The Wilcoxon signed-rank test was used to analyze the EDSS distribution and the improvement of EDSS score defined as "good," "medium," and "poor" between the two groups, while EDSS at attack was adjusted by ordinal logistic regression. Univariable logistic regression analyses were used to identify candidate predictive factors for poor recovery. Independent variables with a $p$ value of $<$ 0.05 in the univariable model or with the clinical relevance were analyzed by multivariate logistic regression. For logistic analysis, "EDSS (acute -6 months post attack)/EDSS (acute - prior to attack)" was used to determine good and poor recovery. The two-tailed $p$ value $<$ 0.05 was considered statistically significant.

\section{Data Availability}

According to consent provided by the participants, any unpublished and anonymized data will be shared from a corresponding author on request from qualified investigators.

\section{Results}

\section{Patients}

We screened 138 individuals for enrollment to the study, of whom 73 were judged to be ineligible or declined to participate. Of the 65 patients who enrolled into the study, $47 \mathrm{pa}-$ tients completed MRI scans twice as required, while 18 completed MRI scan but were excluded based on criteria described in the "Methods" section. Patients were assigned into persistent enhancement and no enhancement groups depending on their MRI images. Of the 44 patients who completed followup at 6 months, 41 were included in the final analysis and 3 were excluded due to relapse within 6 months. All 3 relapsed patients had increased EDSS scores (1.5 to 3.5 points), and the lesions were located in the cervical spinal cord or optic nerve (Fig. 1).

\section{Clinical and Demographic Features}

A total of 41 NMOSD patients were analyzed, of whom 21 $(51.2 \%)$ patients were assigned into the persistent enhancement group and $20(48.8 \%)$ patients were included in the no enhancement group. The sex ratio, median age, disease duration, and ARR were similar between the two groups. A total of $25(61 \%)$ patients were first attack, $9(22 \%)$ were second attack, and the remainder had more than 3 times relapses. The median time of delay prior to acute attack treatment was similar between the PE and NE groups (14 days $v s 13.5$ days, $p=$ 0.168 ). There was no difference in the proportion of patients with AQP4-IgG seropositive between the PE and NE groups (76.2\% vs 85.0\%, $p=0.697$ ). Thirty-three NMOSD attacks were treated with IVMP alone, 4 attacks were treated with IVMP + IVIg, and 4 attacks were treated with IVMP + immunoadsorption (Table 1). Eight patients in each of the $\mathrm{PE}$ and NE groups underwent $\geq 2$ times attacks, and the median EDSS prior to this attack had no statistical difference (1.5 vs $1.75, p=0.873$ ).

\section{MRI Characteristics}

A total of 82 scans for 41 patients, of which 65 were gadolinium-enhancing lesions, were included in the analysis. The majority of lesions were located in the spinal cord in the $\mathrm{PE}$ and NE groups $(80.9 \%$ vs $65 \%, p=0.249)$, while other located in the brainstem $(9.5 \%$ vs $20.0 \%, p=0.697)$, optic 
Fig. 1 Trial profile of the postcontrast MRI scan trial. Gd+ = gadolinium positive; EDSS = Expanded Disability Status Scale; $\mathrm{CNS}=$ central nervous system; $\mathrm{PE}=$ persistent enhancement; $\mathrm{NE}$ $=$ no enhancement.

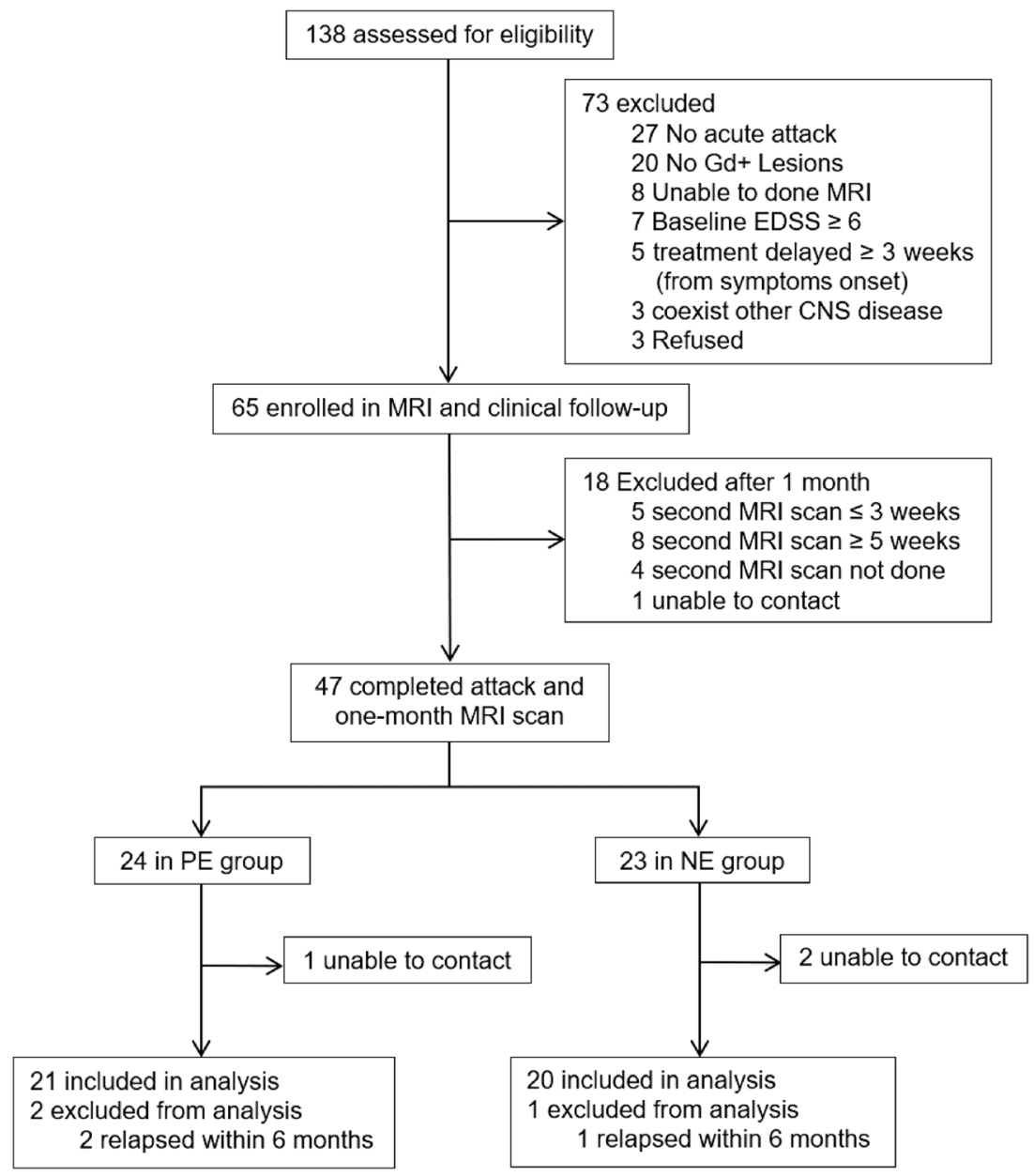

nerve $(4.8 \%$ vs $10 \%, p=0.606)$, and brain $(4.8 \%$ vs $5 \%, p=$ 1.000). The section distribution of spinal cord lesions was similar between the PE and NE groups: cervical, cervicalthoracic, and thoracolumbar (33.3/28.6/19\% vs 40/10/15\%, $p=0.426)$. The distribution of optic nerve lesion, brain lesion, and brainstem lesion was not statistically significant between the PE and NE groups (Table 2). Representative MRI images of both groups are depicted in Fig. 2.

\section{Disability Recovery During 6-Month Follow-Up}

The EDSS ranges from 0 to 10 (divided into 7 groups), with higher scores indicating increased disability. The PE group has a worse distribution of EDSS score (more severe disability) compared with the NE group at attack $(p=0.032)$. We noted the EDSS score improved in both groups, but after adjusting for EDSS at attack, distribution shifts did not show significant differences between the two groups after 1 month (adjusted odds ratio (aOR), 3.589; 95\% CI, 0.918-14.041; $p=0.066$ ). However, patients in the NE group showed a significant shift in the 6-month EDSS distributions compared with those in the PE group (aOR, 10.412; 95\% CI, 2.003-
54.108; $p=0.005)$. We found that patients with persistent enhanced lesions had poor medium-term prognosis (Fig. 3A).

The proportion of "good recovery" was similar between the $\mathrm{PE}$ and NE groups at 1 month $(4.8 \%$ vs $20 \%, p=0.12)$. After 6 months of follow-up, only $14.3 \%$ of the PE group showed a good recovery compared with $50 \%$ of the NE group. Poor recovery rate of the $\mathrm{PE}$ group was higher than that of the $\mathrm{NE}$ group (OR, 3.654; 95\% CI, 1.111-12.001; $p=0.033$ ) (Fig. 3B).

\section{EDSS Change in Patients with AQP4-IgG Positive, First Attack, Longitudinally Extensive Transverse Myelitis, and IVMP Treatment}

In thirty-three patients with AQP4-IgG positive, median EDSS decreased from 4.5 to 3 in the PE group at 6 months compared with 4 to 2 in the NE group. Furthermore, median EDSS of the NE group was lower than that of the PE group after 1 month $(p=0.02)$ and the gap widened between the two groups at 6 months $(p=0.001)$ (Fig. 4).

Twenty-five patients who experienced first attack showed a decrease in median EDSS from 4.0 to 3.5 at 1 month and 2.5 at 6 months in the PE group. By contrast, 
Table 1 Demographic and clinical characteristics of neuromyelitis optica spectrum disorder (NMOSD) patients according to the MRI group

\begin{tabular}{|c|c|c|c|}
\hline & PE group $(n=21)$ & NE group $(n=20)$ & $p$ \\
\hline Age, mean (SD), years & $41.4(16.4)$ & $40.6(16.5)$ & 0.874 \\
\hline Female, $n(\%)$ & $15(71.4)$ & $17(85.0)$ & 0.454 \\
\hline Disease duration, median (IQR), months & $2(0.8-12.0)$ & $1.5(1-8.2)$ & 0.927 \\
\hline Annual relapse rate, mean (SD) & $1.4(1.3)$ & $1.7(1.4)$ & 0.602 \\
\hline First attack, $n(\%)$ & $13(62.0)$ & $12(60.0)$ & 0.901 \\
\hline Second attack, $n(\%)$ & $4(19.0)$ & $5(25.0)$ & 0.719 \\
\hline$\geq 3$ attacks, $n(\%)$ & $4(19.0)$ & $3(15.0)$ & 0.731 \\
\hline Delay from onset to treatment, median (IQR), days & $14(12-20.5)$ & $13.5(8.5-16)$ & 0.168 \\
\hline AQP4-IgG positive, $n(\%)$ & $16(76.2)$ & $17(85.0)$ & 0.697 \\
\hline MOG-IgG positive, $n(\%)$ & $2(9.5)$ & $0(0)$ & 0.488 \\
\hline Qalb $\left(\times 10^{-3}\right)$, median $(\mathrm{IQR})$ & $6.5(4.7-13.1)$ & $5.4(3.9-7.0)$ & 0.069 \\
\hline \multicolumn{4}{|l|}{ Clinical manifestation, $n(\%)$} \\
\hline $\begin{array}{l}\text { Optic neuritis } \\
\text { LETM }\end{array}$ & $\begin{array}{l}1(4.8) \\
17(80.9)\end{array}$ & $\begin{array}{l}2(10.0) \\
13(65.0)\end{array}$ & \multirow[t]{3}{*}{0.717} \\
\hline Brain & $1(4.8)$ & $1(5.0)$ & \\
\hline Brainstem & $2(9.5)$ & $4(20.0)$ & \\
\hline Coexisting autoimmune diseases, $n(\%)$ & $8(38.1)$ & $3(15.0)$ & 0.093 \\
\hline \multicolumn{4}{|l|}{ Treatment, $n(\%)$} \\
\hline $\begin{array}{l}\text { IVMP } \\
\text { IVMP + IVIg }\end{array}$ & $\begin{array}{l}16(76.2) \\
2(9.5)\end{array}$ & $\begin{array}{l}17(85.0) \\
2(10.0)\end{array}$ & \multirow[t]{2}{*}{0.84} \\
\hline IVMP + IA & $3(14.3)$ & $1(5.0)$ & \\
\hline \multicolumn{4}{|l|}{ Relapse prevention treatment, $n(\%)$} \\
\hline $\begin{array}{l}\text { Oral prednisone }+ \text { RTX } \\
\text { Oral prednisone }+ \text { MMF }\end{array}$ & $\begin{array}{l}8(38.1) \\
1(4.8)\end{array}$ & $\begin{array}{l}6(30.0) \\
2(10.0)\end{array}$ & \multirow[t]{3}{*}{0.328} \\
\hline Oral prednisone & $12(57.1)$ & $9(45.0)$ & \\
\hline Not use & $0(0)$ & $3(15.0)$ & \\
\hline
\end{tabular}

$\mathrm{PE}=$ persistent enhancement; $\mathrm{NE}=$ no enhancement; $\mathrm{SD}$ = standard deviation; IQR = inter-quartile range; $\mathrm{AQP} 4$ = aquaporin-4; MOG = myelin oligodendrocyte glycoprotein; Qalb = CSF albumin/serum albumin; $\mathrm{LETM}=$ longitudinally extensive transverse myelitis; IVMP = intravenous methylprednisolone therapy; IVIg = intravenous immunoglobulin; IA = immunoadsorption; RTX = rituximab; MMF = mycophenolate mofetil. the median EDSS score for patients in the NE group dropped from 3.5 to 2 at 6 months. In patients with first episode, the improvement of EDSS in the NE group after 1-month follow-up was significant compared with that in
Table 2 MRI characteristics of the PE and NE groups

\begin{tabular}{llll}
\hline & PE group $(n=21)$ & NE group $(n=20)$ & $p$ \\
\hline Optic nerve & & & \\
$\quad$ Unilateral, $n(\%)$ & 0 & $2(10.0)$ & 0.333 \\
Optic chiasm, $n(\%)$ & $1(4.8)$ & & \\
Spinal cord & & & \\
Cervical, $n(\%)$ & $7(33.3)$ & $2(40.0)$ & 0.426 \\
Cervical-thoracic, $n(\%)$ & $6(28.6)$ & $3(15.0)$ & \\
Thoracolumbar, $n(\%)$ & $4(19.0)$ & & \\
Cerebral & & $1(5.0)$ & 1.000 \\
Periventricular white matter, $n(\%)$ & $1(4.8)$ & $1(5.0)$ & \\
Cortex and subcortical white matter, $n(\%)$ & $1(4.8)$ & $3(15.0)$ & \\
Brainstem & & $3(15.0)$ & \\
Area postrema, $n(\%)$ & $2(9.5)$ & & \\
Pontine, $n(\%)$ & $1(4.75)$ & & \\
\hline
\end{tabular}

$\mathrm{PE}=$ persistent enhancement $\mathrm{NE}=$ no enhancement 
Fig. 2 Representative gadolinium enhancement MRI images of NMOSD patients. (A) Patchy gadolinium-enhanced lesion was found in $\mathrm{C} 1-3$ and persistently existed after 1 month. (B) Openring enhanced lesion in $\mathrm{C} 1-3$ disappeared at 1-month follow up.

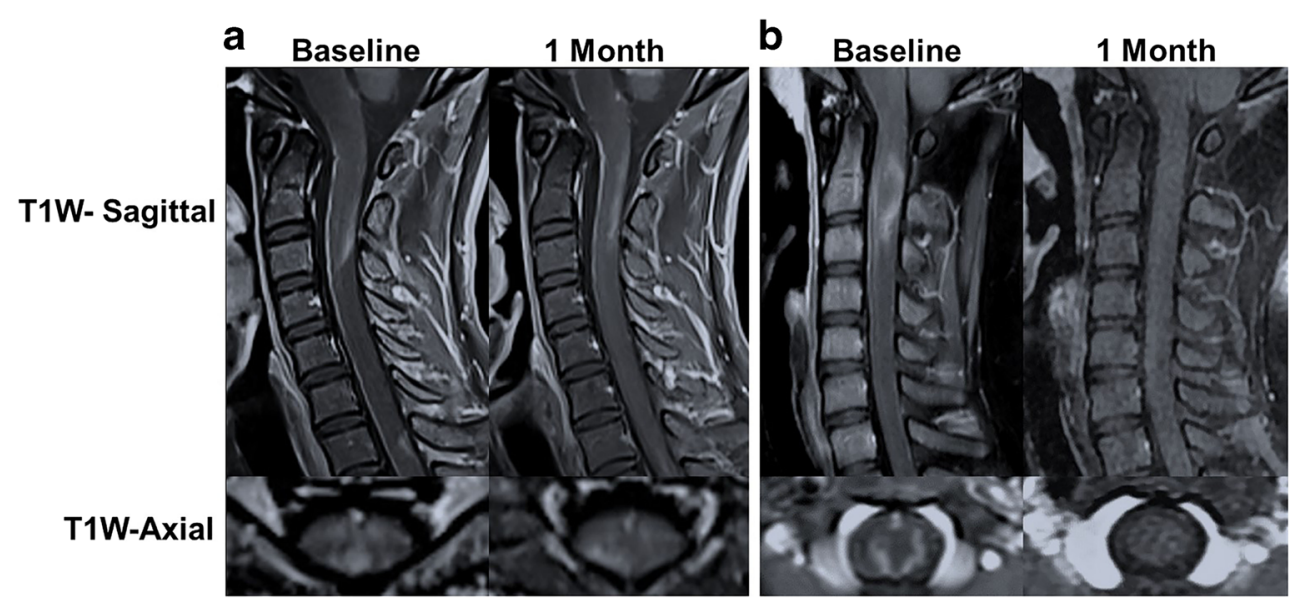

the PE group $(p=0.032)$, and the trend continued to 6 months $(p=0.042)$ (Fig. 4).

Disability improved in 30 patients with longitudinally extensive transverse myelitis (LETM) during follow-up. Median EDSS decreased from 4 to 3.5 at 1 month and further to 3 at 6 months in the PE group. Correspondingly, disability measured by EDSS improved from 3 to 2 points after 1-month follow-up and remained 2 at 6 months in the NE group. Compared with that in the PE group, a lower median EDSS was observed in the NE group after 1 month $(p=0.034)$ and the trend continued to 6 months ( $p=0.001)$ (Fig. 4).

In 33 patients treated only with IVMP, median EDSS decreased from 4 to 3 in the PE group compared with 3.5 to 2 in the NE group at 6 months. Median EDSS of the NE group was lower than that of the PE group after 1 month $(p=0.027)$, and the gap between the two groups was even more pronounced at 6 months $(p=0.003)$ (Fig. 4).

\section{Predictive Factors for Medium-Term Recovery}

Multivariate logistic regression (MLR) analysis was used to assess the predictive factors of recovery in NMOSD patients. MLR analysis of lesions, AQP4-IgG positive, first attack, and Qalb showed that only persistently enhancing lesions (OR, 5.473; 95\% CI, 1.417-21.115; $p=0.014)$, first attack (OR, $0.226 ; 95 \% \mathrm{CI}, 0.059-0.862 ; p=0.029)$ were significant predictive factors (Table 3).

\section{Discussion}

This study investigated the impact of persistent gadoliniumenhancing lesions on the prognosis of NMOSD attack. We found that physical disability improvement in the PE group was less than that in the NE group at 1 month, and the trend a

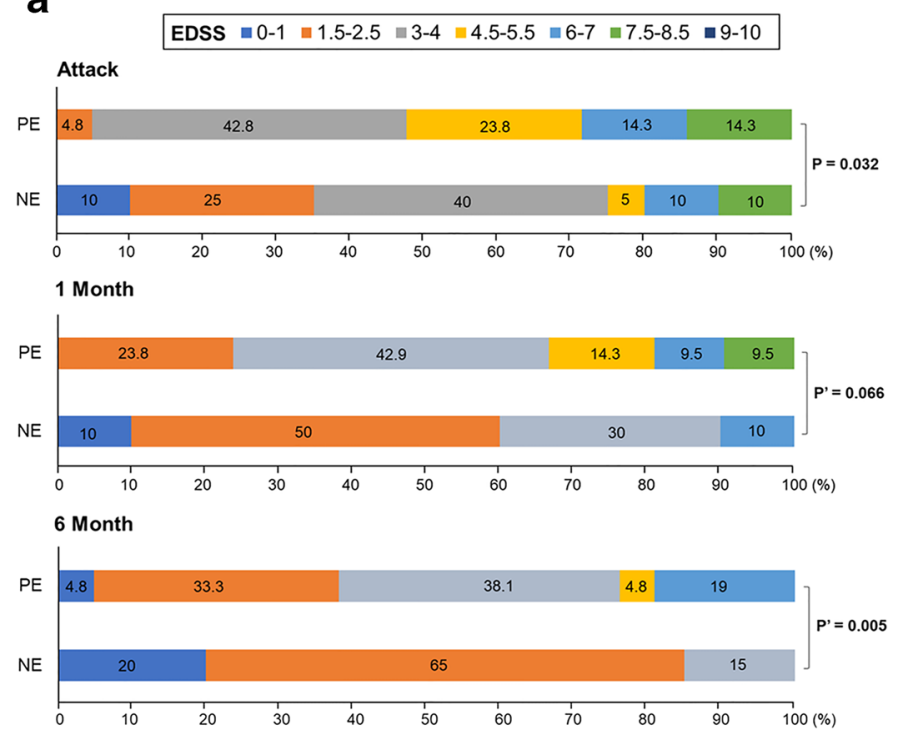

b

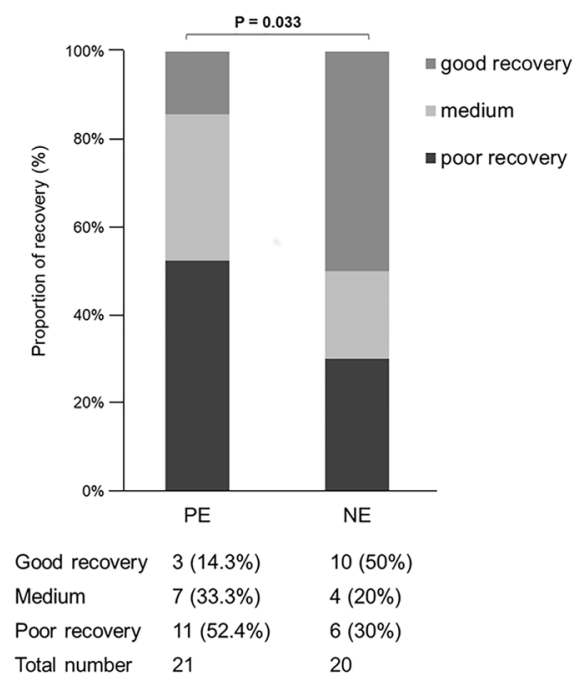

Fig. 3 The changes of EDSS score in the PE and NE groups after followup. (A) Distribution of Expanded Disability Status Scale (EDSS) scores at attack, at 1 month and 6 months in both groups. (B) The improvement of
EDSS score in the persistent enhancement group was worse than that in the no enhancement group at 6 months. $p^{\prime}=$ adjusted $p$ value. 

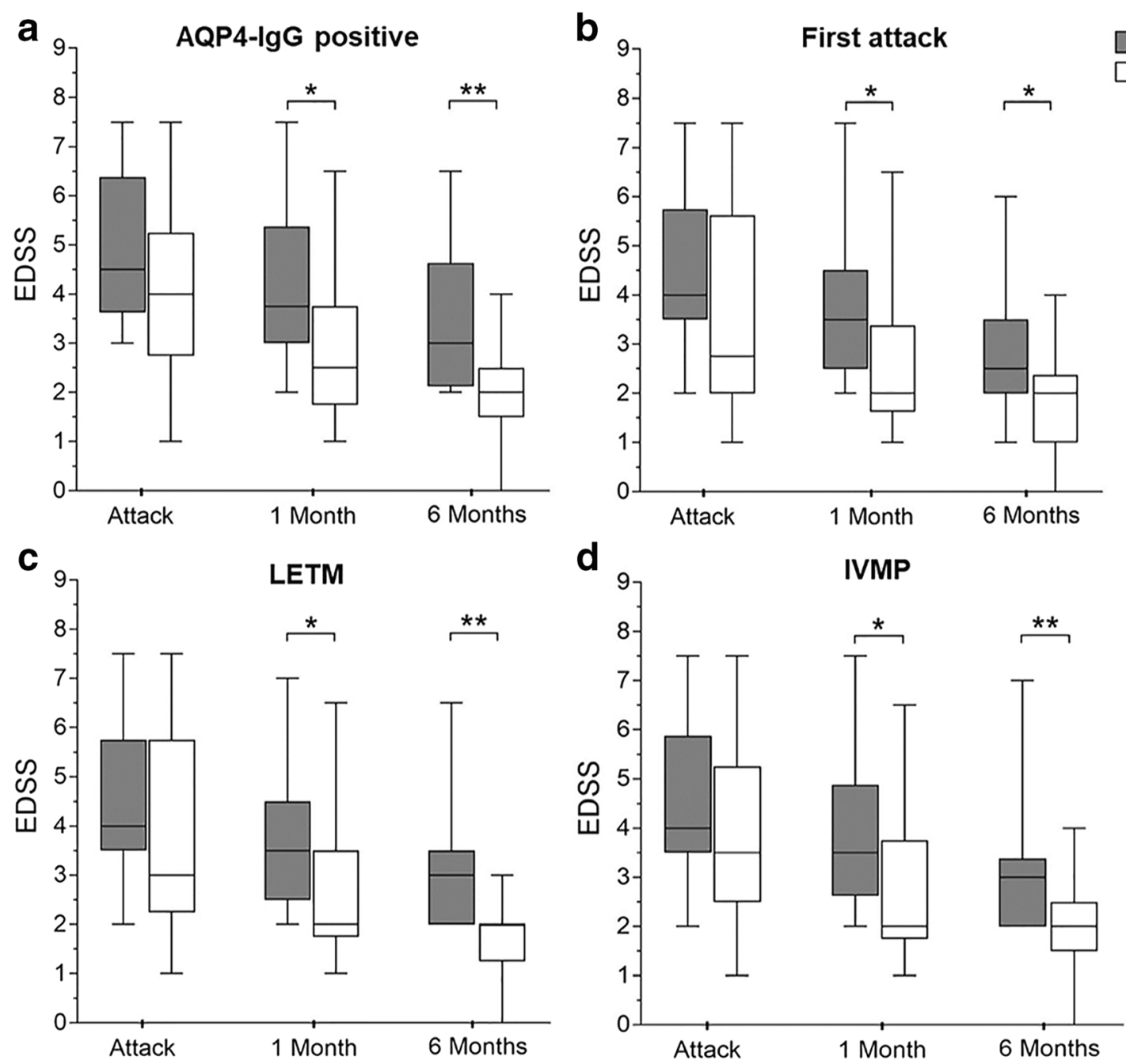

Fig. 4 Comparison of EDSS score changes in patients with AQP4-IgG positive, first attack, LETM, and IVMP treatment. (A) Median EDSS score of the NE group was lower in AQP4-IgG-positive patients at 6 month $(n=33)$. (B) EDSS improvement in the PE group was less than that in the NE group in patients who experienced first attack $(n=25)$. (C)

In patients with transverse myelitis, EDSS improved poorly in the PE group compared with the NE group $(n=30)$. (D) In patients treated only with IVMP, 6-month median EDSS score was lower in the NE group $(n=33) . * p<0.05$ and $* * p<0.01$.

Table 3 Univariate and multivariate logistic regression analyses of prognosis-predictive factors in neuromyelitis optica spectrum disorder (NMOSD) attack

\begin{tabular}{|c|c|c|c|c|}
\hline & \multicolumn{2}{|l|}{ Univariate analysis } & \multicolumn{2}{|l|}{ Multivariate analysis } \\
\hline & OR $(95 \% \mathrm{CI})$ & $p$ value & OR $(95 \% \mathrm{CI})$ & $p$ value \\
\hline Age & $1.003(0.968-1.038)$ & 0.878 & - & - \\
\hline Sex, male & $2.415(0.601-9.718)$ & 0.214 & - & - \\
\hline Annual relapse rate & $1.404(0.893-2.203)$ & 0.141 & - & - \\
\hline Persistently enhancing lesions & $3.654(1.112-12.001)$ & $0.033^{*}$ & $5.473(1.417-21.115)$ & $0.014 *$ \\
\hline First attack & $0.271(0.080-0.913)$ & $0.035^{*}$ & $0.226(0.059-0.862)$ & $0.029 *$ \\
\hline AQP4-IgG positive & $0.655(0.153-2.801)$ & 0.569 & $0.823(0.162-4.178)$ & 0.814 \\
\hline Delay from onset to treatment & $0.912(0.803-1.034)$ & 0.152 & - & - \\
\hline Qalb & $1.015(0.924-1.116)$ & 0.751 & $1.073(0.956-1.203)$ & 0.225 \\
\hline Relapse prevention treatment & $0.787(0.140-4.419)$ & 0.786 & - & - \\
\hline
\end{tabular}

Qalb = CSF albumin/serum albumin; $\mathrm{OR}=$ odds ratio; $\mathrm{CI}=$ confidence interval.

${ }^{*} p<0.05$ representing statistical significance. 
continued to 6 months. In patients with AQP4-IgG positive, first attack, LETM, or IVMP treatment, median EDSS of the NE group was lower than that of the PE group after 6-month follow-up. Furthermore, we have confirmed that the persistent existence of gadolinium-enhancing lesions is a predictor of poor prognosis in NMOSD attack.

All NMOSD patients in our cohort were experiencing acute attack with enhanced lesions. The presence of gadoliniumenhancing lesions indicates that contrast agent has entered the CNS via a compromised BBB, as gadolinium compounds do not readily cross a stable BBB [16]. Hence, enhancing lesions of demyelinating patients reflects the destruction of the BBB $[17,18]$. Several signs on activity and persistence of lesions have been obtained in vivo in NMOSD patients by contrast MRI. In our series of NMOSD patients, enhancement was displayed in poorly marginated and multiple patchy patterns mainly in the lateral ventricle, the optic nerve, or the cervical and upper thoracic medulla. The enhanced lesions described above (so-called "cloud-like" enhancement of brain lesions), chiasmal enhancement of optic neuritis, and ring enhancement of the spinal cord are more common in NMOSD patients $[19,20]$.

Changes in the distribution of EDSS score is significantly decreased in the PE group than in the NE group at 1 month and 6 months, reflective of slower improvement. Meanwhile, the proportion of good recovery in the PE group was lower than that in the NE group. Persistent disruption of the BBB may be the pathological mechanism for poor prognosis of patients in the PE group. The BBB is a crucial barrier that limits brain entry of peripheral cells and potentially harmful molecules. The persistent existence of gadolinium-enhancing lesions indicates that the BBB is still open, and peripheral AQP4 antibodies and inflammatory factors may still enter the lesions exacerbating damage [21]. Inflammatory mediators can disrupt the BBB, allowing AQP4 antibodies or other inflammatory factors to enter into the CNS and initiate the pathological process of NMOSD [22]. As disability improved, only $55.43 \%$ of new enhancing lesions gradually disappear within 3 weeks [23]. The increased BBB permeability is an important feature of NMOSD patients, therefore a crucial assessment during disease progression. The level of matrix metalloproteinase-2/9 (MMP-2/9) is a confirmed biomarker of BBB disruption [24, 25]; however, lumbar puncture is invasive and patients are less compliant to dynamic continuous screening. Dynamic contrast-enhanced MRI (DCE-MRI) provides semi-quantitative and quantitative data to accurately assess the permeability of the BBB [26], but it has the disadvantage of long scanning time $(15 \mathrm{~min})$ and the requirement of a unique post-process method. By contrast, enhanced MRI is a reliable and convenient method of detecting BBB breakdown.

Additionally, several other factors were assessed for their impact on the prognosis of NMOSD patients. Subgroup analysis showed that AQP4-IgG-positive patients seem to experience more severe clinical attacks and have a higher relapse rate [27]. Meanwhile, worse recovery from the first attack is a predictor of disability, accumulating to disability of previous attack with pronounced impact on prognosis assessment [28]. In addition, EDSS score seems to be more sensitive to assessment of LETM-related dysfunction [29]. More importantly, IVMP is still the first-line option for most patients with acute episodes [1]. Therefore, it is necessary to analyze the prognosis of patients in AQP4-IgG-positive, first attack, LETM, or IVMP treatment subgroup.

We analyzed the AQP4-IgG subgroup and still found a trend for better recovery in the NE group. Subgroup analysis of first-episode patients showed that patients in the PE group had more severe disabilities at 6 months. High-dose steroids is the mainstay therapy for acute relapses targeting cell mediate inflammation, while PLEX or IA appears to be very promising in elimination of specific antibodies and complement and proinflammatory factors [30, 31]. Moreover, the benefit of early initiation of PLEX/IA was confirmed rather than its relevance as a rescue therapy after steroid failure $[14,31]$. However, due to few patients treated by IA in our study, the subgroup analysis was performed only for all IVMP treatments. EDSS improved poorly in patients treated with IVMP from the PE group than the NE group. Furthermore, LETM presented with markedly higher EDSS scores than brain-dominant type in patients with NMOSD [32]. Here, LETM was characterized by symptoms and signs of neurologic dysfunction in motor and sensory tracts on both sides of the spinal cord. Using EDSS to measure disability in LETM is more accurate, since it is sensitive to assess physical disabilities [29]. In LETM subgroup analysis, the PE group experienced poorer improvement in disability compared with the NE group. Patients with first attack tend to have favorable outcome, and our analysis of patients with first attack also found better prognosis in the NE group.

We found that a persistently gadolinium-enhancing lesion is an important factor in prognosis prediction of NMOSD attack. It is important to closely follow-up NMOSD patients with acute attacks and adjust or escalate treatment timely [33, 34]. Furthermore, most patients with NMOSD attack have only $19.1 \%$ complete remission rates after the first course of treatment, which may be associated with lack of adequate short-term follow-up [5]. There are several MRI imaging features, including T1 hypointensity, cavitation, or atrophy, indicatives that patients with NMOSD are more likely to have a high risk of poor recovery and permanent disability $[19,35]$. Usually, most of these predictors reflect chronic changes of disease and cannot provide more information for effective therapy escalation. Thus, it becomes important to assess BBB function in acute phase by contrast MRI.

Our study has several limitations. First, the present study was limited by small sample size, and future high-quality research with bigger sample size is required to confirm the 
conclusions herein presented. Moreover, we did not evaluate neurofilament protein, glial fibrillary acidic protein, or cytokines in serum or CSF since this was beyond the scope of our current study. Finally, it should be noted that our current study does not provide quantitative assessment of BBB permeability, but we have gradually carried out DCE-MRI studies that can more accurately assess BBB leakage.

\section{Conclusion}

Our data suggest that the presence of persistently gadoliniumenhancing lesions might be useful in determining mediumterm poor recovery after NMOSD attack and that more intensive treatment or immunosuppressive therapy might be warranted if the patient has persistently gadolinium-enhancing lesions.

Supplementary Information The online version contains supplementary material available at https://doi.org/10.1007/s13311-020-00973-9.

Acknowledgments We thank our patients for participating in this study and all the members of Jing-Jin Neuroimmunology Teams for recruiting patients and other support.

Required Author Forms Disclosure forms provided by the authors are available with the online version of this article.

Authors' Contributions D.C.T. and X.Z. formulated the conception and design of this study; X.L. and W.X. contributed to the acquisition and analysis of clinical data; X.W. and Y.D. contributed to the acquisition and analysis of MRI data; Y.L. and Y.R. made a critical revision; Y.X drafted the manuscript and prepared the figures.

Funding Work in the authors' laboratories was supported in part by grants from the National Natural Science Foundation of China (81801199).

Data Availability The datasets used or analyzed during the current study are available from the corresponding author on reasonable request.

\section{Compliance with Ethical Standards}

Competing interests The authors declare that they have no competing interests.

Ethical Approval and Consent to Participate This project was approved by the Ethics Committee of Beijing Tiantan Hospital, Capital Medical University, Beijing, China (KY 2019-050-02).

Consent for Publication Not applicable.

\section{References}

1. Weinshenker BG, Wingerchuk DM. Neuromyelitis Spectrum Disorders. Mayo Clin Proc 2017;92(4):663-79.
2. Lennon VA, Wingerchuk DM, Kryzer TJ, et al. A serum autoantibody marker of neuromyelitis optica: distinction from multiple sclerosis. Lancet 2004;364(9451):2106-12.

3. Collongues N, Ayme-Dietrich E, Monassier L, de Seze J. Pharmacotherapy for Neuromyelitis Optica Spectrum Disorders: Current Management and Future Options. Drugs. 2019;79(2): $125-42$.

4. Elsone L, Panicker J, Mutch K, et al. Role of intravenous immunoglobulin in the treatment of acute relapses of neuromyelitis optica: experience in 10 patients. Mult Scler 2014;20(4):501-4.

5. Kleiter I, Gahlen A, Borisow N, et al. Neuromyelitis optica: Evaluation of 871 attacks and 1,153 treatment courses. Ann Neurol 2016;79(2):206-16.

6. Seok JM, Cho HJ, Ahn SW, et al. Clinical characteristics of lateonset neuromyelitis optica spectrum disorder: A multicenter retrospective study in Korea. Mult Scler 2017;23(13):1748-56.

7. Takano R, Misu T, Takahashi T, et al. Astrocytic damage is far more severe than demyelination in NMO: a clinical CSF biomarker study. Neurology. 2010;75(3):208-16.

8. Bergamaschi R, Ghezzi A. Devic's neuromyelitis optica: clinical features and prognostic factors. Neurol Sci 2004;25 Suppl 4:S3647.

9. Shimizu F, Nishihara H, Kanda T. Blood-brain barrier dysfunction in immuno-mediated neurological diseases. Immunol Med 2018;41(3):120-8

10. Uchida $\mathrm{T}$, Mori M, Uzawa A, et al. Increased cerebrospinal fluid metalloproteinase- 2 and interleukin- 6 are associated with albumin quotient in neuromyelitis optica: Their possible role on blood-brain barrier disruption. Mult Scler 2017;23(8):1072-84.

11. Waubant E. Biomarkers indicative of blood-brain barrier disruption in multiple sclerosis. Dis Markers 2006;22(4):235-44.

12. Wingerchuk DM, Banwell B, Bennett JL, et al. International consensus diagnostic criteria for neuromyelitis optica spectrum disorders. Neurology. 2015;85(2):177-89.

13. Kaiser PK. Prospective evaluation of visual acuity assessment: a comparison of snellen versus ETDRS charts in clinical practice (An AOS Thesis). Trans Am Ophthalmol Soc 2009;107:311-24.

14. Bonnan M, Valentino R, Debeugny S, et al. Short delay to initiate plasma exchange is the strongest predictor of outcome in severe attacks of NMO spectrum disorders. J Neurol Neurosurg Psychiatry 2018;89(4):346-51.

15. Freedman MS, Thompson EJ, Deisenhammer F, et al. Recommended standard of cerebrospinal fluid analysis in the diagnosis of multiple sclerosis: a consensus statement. Arch Neurol 2005;62(6):865-70.

16. Albrecht DS, Granziera C, Hooker JM, Loggia ML. In Vivo Imaging of Human Neuroinflammation. ACS Chem Neurosci 2016;7(4):470-83.

17. Grossman RI, Braffman BH, Brorson JR, et al. Multiple sclerosis: serial study of gadolinium-enhanced MR imaging. Radiology. 1988;169(1):117-22.

18. Katz D, Taubenberger JK, Cannella B, et al. Correlation between magnetic resonance imaging findings and lesion development in chronic, active multiple sclerosis. Ann Neurol 1993;34(5):661-9.

19. Kim HJ, Paul F, Lana-Peixoto MA, et al. MRI characteristics of neuromyelitis optica spectrum disorder: an international update. Neurology. 2015;84(11):1165-73.

20. Yokote H, Nose Y, Ishibashi S, et al. Spinal cord ring enhancement in patients with neuromyelitis optica. Acta Neurol Scand 2015;132(1):37-41.

21. Obermeier B, Daneman R, Ransohoff RM. Development, maintenance and disruption of the blood-brain barrier. Nat Med 2013;19(12):1584-96.

22. Guo Y, Weigand SD, Popescu BF, et al. Pathogenic implications of cerebrospinal fluid barrier pathology in neuromyelitis optica. Acta Neuropathol 2017;133(4):597-612. 
23. Cotton F, Weiner HL, Jolesz FA, Guttmann CR. MRI contrast uptake in new lesions in relapsing-remitting MS followed at weekly intervals. Neurology. 2003;60(4):640-6.

24. Tomizawa Y, Yokoyama K, Saiki S, et al. Blood-brain barrier disruption is more severe in neuromyelitis optica than in multiple sclerosis and correlates with clinical disability. J Int Med Res 2012;40(4):1483-91.

25. Hosokawa T, Nakajima H, Doi Y, et al. Increased serum matrix metalloproteinase-9 in neuromyelitis optica: implication of disruption of blood-brain barrier. J Neuroimmunol 2011;236(1-2):81-6.

26. Cramer SP, Modvig S, Simonsen HJ, Frederiksen JL, Larsson HB. Permeability of the blood-brain barrier predicts conversion from optic neuritis to multiple sclerosis. Brain. 2015;138(Pt 9):2571-83.

27. Jarius S, Ruprecht K, Wildemann B, et al. Contrasting disease patterns in seropositive and seronegative neuromyelitis optica: A multicentre study of 175 patients. J Neuroinflammation 2012;9:14.

28. Seok JM, Cho EB, Lee HL, et al. Clinical characteristics of disabling attacks at onset in patients with neuromyelitis optica spectrum disorder. J Neurol Sci 2016;368:209-13.

29. Meyer-Moock S, Feng YS, Maeurer M, Dippel FW, Kohlmann T. Systematic literature review and validity evaluation of the Expanded Disability Status Scale (EDSS) and the Multiple Sclerosis Functional Composite (MSFC) in patients with multiple sclerosis. BMC Neurol 2014;14:58.
30. Abboud H, Petrak A, Mealy M, et al. Treatment of acute relapses in neuromyelitis optica: Steroids alone versus steroids plus plasma exchange. Mult Scler 2016;22(2):185-92.

31. Lipphardt M, Muhlhausen J, Kitze B, et al. Immunoadsorption or plasma exchange in steroid-refractory multiple sclerosis and neuromyelitis optica. J Clin Apher 2019;34(4):381-91.

32. Nagaishi A, Takagi M, Umemura A, et al. Clinical features of neuromyelitis optica in a large Japanese cohort: comparison between phenotypes. J Neurol Neurosurg Psychiatry 2011;82(12): 1360-4.

33. Kimbrough DJ, Fujihara K, Jacob A, et al. Treatment of Neuromyelitis Optica: Review and Recommendations. Mult Scler Relat Disord 2012;1(4):180-7.

34. Sellner J, Boggild M, Clanet M, et al. EFNS guidelines on diagnosis and management of neuromyelitis optica. Eur J Neurol 2010;17(8): 1019-32.

35. Cassinotto C, Deramond H, Olindo S, et al. MRI of the spinal cord in neuromyelitis optica and recurrent longitudinal extensive myelitis. J Neuroradiol 2009;36(4):199-205.

Publisher's Note Springer Nature remains neutral with regard to jurisdictional claims in published maps and institutional affiliations. 